\title{
Periodic Table Gets Crowded In Year 2011.
}

\author{
P.R.Shukla
}

\begin{abstract}
Year 2011, has been specially important for teachers and students of chemistry, as after a gap of about 14 years at least five new elements were named and included in the periodic table. All these elements are synthetic and radioactive and some were actually made in 1999, but got their name and status by IUPAC, in July 2011. The total number of elements now in periodic table is 112, and scientists are trying their best to prepare elements with atomic numbers 118,119 and 120 as well.
\end{abstract}

In recent past, the attention of media seems to have shifted from reporting news of politics, health, movies and fitness, to the new discoveries being made in laboratories. A number of new ideas have been put forward which still need confirmation, but have captured reader's attention. The year 2011 has been specially interesting for teachers and students of chemistry, because of the inclusion of several new elements in the Periodic Table (P.T) in November. This is important, as after 1997 ( a gap of 14 years), such large scale naming and status allocation to the elements in the P.T has been wintnessed.

All material things in the world are made up of different combination of about 100 or more elements, the latter being a substance incapable of being broken down or changed into another substance by chemical means and has characterstic physical and chemical properties. In 1870 after more than 60 elements had been identified, a Russian scientist Dmitri Ivanovich Mendleev while writing his book "Principles of chemistry", felt the need of a systematic arrangement of elements and thus created the periodic table (P.T) divided in periods and groups, in which the elements were arranged in the increasing order of their atomic weights. In order that elements of similar chemical properties appeared in the same group of (P.T), he left some blanks in the table, with the prediction, that these will be filled up later by the discovery of new elements with similar properties. Very soon, his prediction, was found correct with the discovery of Gallium in 1875 in France, Scandium in 1879 in Scandinavia and Germanium in 1886 in Germany. But soon the concept of atomic weight appeared erroneous as the atomic weight of Argon placed it in $1^{\text {st }}$ group along with Sodium and Potassium (alkali metals) though it is an inert gas. In 1913 H.Mosley, came up with the idea of arranging the elements in the increasing order of their atomic numbers $\mathrm{Z}$, (the sum of protons and neutrons in the nucleus of the atom). This number is unique for every element and decides its behaviour. This is the modern version of P.T in use till now.

In the nineteenth century, some more elements were discovered, and the last element of the table being Lawrencium, a member of transactinide series $\left({ }_{89} A c-{ }_{103} \mathrm{Lr}\right)$. The elements with $\mathrm{Z}=104-109$, were discovered jointly at the Lawrence Berkley National Laboratory (LBNL), California, U.S.A and the Joint Institute for Nuclear Research (JINR) at Dubna, Russia in 1990. Their official names and status were however, given much later and after several years of controversy. On March 6,1997, the New York times, published the existence and names of these elements as accepted by the nucleur scientists and approved by IUPAC which are (i) Rutherfordium, Rf (104), (ii) Dubnium, Db (105), Seaborgium, Sg (106), Bohrium, Bh (107), Hassium, Hs, (108) and Meitnerium, Mt (109). The Russians had however, proposed the name Kurchatovium,Ku, for element number 104, but the IUPAC agreed to the above name Rutherfordium, given by researchers at Berkley.The name of element 106 is Seaborgium, in honour of Glen.T. Seaborg, who discovered it.

It was also found that the elements with their $\mathrm{Z}$ values higher than 104, are;

(a) radioactive i,e too unstable to exist in nature, and decay rapidly into other elements (IUPAC defines that for an element to be stable and to exist, it's life time should be greater than $10^{-4}$ seconds, which is the time a nucleus takes to form a radioactive cloud) and,

(b) super heavy and as such do not occur in nature and can be created only in the laboratory and that too with difficulty using the technique of nuclear fusion.

In 1997, the criteria for the claim of discovery of an element was put forward very clearly by the 'IUPAC/ IUPAP Transfermium group', as "Discovery of a chemical element is the experimental demonstration beyond reasonable doubt of the existence of a nucleotide". The official names and positions of the elements in the P.T were decided much later. The name of the element is preferably given in the honor of the city, laboratory or scientist, who has been associated with it's discovery and both these have to be ratified by several scientific bodies before the final decision is taken and an announcement is made. Several times, the existence of the element is accepted by scientific bodies, but not its name. In such cases the element is named according to the old method of nomenclature.

After the discovery of Meitnerium with $\mathrm{Z}=109$, during the next 14 years, two other laboratories in 
addition to upper two, with assess to unique materials and techniques, required for these fusion experiments, also engaged in synthesis of the elements. These are:

(i) Lawrence Berkley National Laboratory (LBNL) California, U.S.A, which has the credit of successfully discovering elements (104-109) with the help of their long standing heavy element group,

and, (ii) Gesellschaft fur Schwerionenforschung or Institute of heavy ion research at Dermstadt, Germany (GSI). The discovery of the elements, their nomenclature and position in the PT is now being given.

\section{( 1) Elements 110 -112}

(a) Discovery; On November 9,1994, the first atom of ' 110 ', was claimed to have been prepared by (GSI) in Darmstadt and later a joint collaboration of LBNL and JINR, on Feb 19,1996, also created 273 isotope of the element by fusing ${ }_{82} \mathrm{~Pb}^{208}$ and ${ }_{28} \mathrm{Ni}^{64}$ but this decays to ${ }_{104} \mathrm{Rf}^{257}+{ }_{103} \mathrm{No}^{253}$.

The 272 isotope of ' 111 ' with half life of $\approx 26$ seconds was prepared by GSI on December 8,1996 and the Hoffmann and Dubna group reconfirmed this by preparing 273 isotope on May 6, 2002, by the reaction of ${ }_{83} \mathrm{Bi}^{209}$ and ${ }_{28} \mathrm{Ni}^{64}$. The decay product in case of both isotopes is ${ }_{105} \mathrm{Db}^{282}$ and ${ }_{103} \mathrm{Lr}^{256}$.

In 2002, the Hoffmann and Dubna group, claimed to have obtained ' 112 ' by fusing ${ }_{30} \mathrm{Zn}^{70}$ with ${ }_{82} \mathrm{~Pb}^{208}$, and this reaction was confirmed in 2004, by Japanese researchers at RIKEN. The only decay product identified was ${ }_{106} \mathrm{Sg}^{265}$. On May 19, 2009, the IUPAC, however, gave the credit of discovery to the Hoffmann group at LBNL,U.S.A.

(b) Nomenclature; The names of all the elements were approved by IUPAC, in November 11,2011. ' 110 ' and ' 111 ' were named in honor of the GSI Helmholz center for heavy ion research at Darmstad, Germany and the noble prize winning physicist Wilhelm Conrad Roentgen of the same institute as Darmstadtium (Ds) and Roentgenium, $(\mathbf{R g})$ respectively. '112' was named as Copernicium $(\mathbf{C n})$, after the famous astronomer Nicolas Copernicus. These names were announced in June, 2012.

(c) Position in table; All the three elements are present in period seven and occupy $10^{\text {th }}, 11^{\text {th }}$ and $12^{\text {th }}$ groups. Their electronic configurations are ;

Ds- $-[\mathrm{Rn}] 5 \mathrm{f}^{14} 6 \mathrm{~d}^{9} 7 \mathrm{~s}^{1}$

$\mathrm{Rg}-[\mathrm{Rn}] 5 \mathrm{f}^{14} 6 \mathrm{~d}^{10} 7 \mathrm{~s}^{1}$

$\mathrm{Cn}-[\mathrm{Rn}] 5 \mathrm{f}^{14} 6 \mathrm{~d}^{10} 7 \mathrm{~s}^{2}$

Thus only Ds is a truly d-block metal but $\mathrm{Rg}$ and Cn have completely filled d-orbitals and in behavior resemble the other metals of their groups as $\{\mathrm{Cu}, \mathrm{Ag}, \mathrm{Au}\}$ and $\{\mathrm{Zn}, \mathrm{Cd}, \mathrm{Hg}\}$.

\section{(11) Elements 115 \& 113:}

\section{(a) Discovery;}

The elements with odd $\mathrm{Z}$ values are superheavy, conventionally very unstable and more difficult to prepare. The isotopes $287 \& 287$ of ' 115 ' were prepared by the joint collaboration of LLNL, U.S.A. and JINR, Dubna, Russia. The experiment was started on July 14, and ended on August 3, 2003 and the following reaction takes place ;

${ }_{95} \mathrm{Am}^{209}+{ }_{20} \mathrm{Ca}^{48}-115^{288}$ and $115^{287}$

The nuclei have half life of 90 millisec and sequence of decay by the emission of alpha particles is;

' 115 ' - '113' - '111' - ${ }_{105} \mathrm{Db}$.

In 2006, the discovery of 274 isotope of ' 113 ' was also claimed by RIKEN, Japan by fusion of Bi and Zn nuclei, as shown below;

${ }_{83} \mathrm{Bi}^{209}+{ }_{30} \mathrm{Zn}^{70}-113^{273}-111^{274}+{ }_{109} \mathrm{Mt}^{270}$

The IUPAC has not yet accepted the claim of existence of any of these elements and has required more convincing evidences.

(b) Nomenclature and Position in table ; As the very existence of the elements is not accepted naming of these is not possible. These are therefore known by their old names, Ununtrium (Uut) and Ununpentium (Uup) for ' 113 ' and ' 115 ' respectively for the time being. The elements appear in the P.T, by the above names. These are hypothetically placed in seventh period. The former is in group 13 with co-members $\{\mathrm{B}, \mathrm{Al}, \mathrm{Ga}$, In and $\mathrm{Tl}\}$ and is also called ekathallium and ' 115 ' is in group 15 with co-members $\{\mathrm{N}, \mathrm{P}, \mathrm{As}, \mathrm{Sb}$ and $\mathrm{Bi}\}$ and called ekabismuth. As in case of both these groups, the metallic character increases as one goes down the group, these should behave as metals, although both are p-block. Their suggested electronic configurations are;

Uut - [Rn] $5 \mathrm{f}^{14} 6 \mathrm{~d}^{10} 7 \mathrm{~s}^{2} 7 \mathrm{p}^{1}$

Uup - $[$ Rn $] 5 f^{14} 6 d^{10} 7 s^{2} 7 p^{3}$ 
(111) Elements 114 \& 116:

(a) Discovery: Both these are also super heavy, and their stablest isotopes as 289 and 292 respectively were prepared in August 2003, under the joint collaboration of LLNL,California, U.S.A and JINR, Dubna, Russia by the fusion of $\mathrm{Pu}(\mathrm{Cm})$ and $\mathrm{Ca}$ nuclei as shown below;

${ }_{94} \mathrm{Pu}^{244}+{ }_{20} 20 \mathrm{Ca}^{48}-114^{289}$, with a half life of $30 \mathrm{sec}$, which decays at once as;

${ }_{112} \mathrm{Cn}^{281}-(1 / 5 \mathrm{sec}){ }_{110} \mathrm{Ds}^{277}-(0.08 \mathrm{sec}){ }_{108} \mathrm{Hs}^{273}-(1 / 3 \mathrm{sec}){ }_{106} \mathrm{Sg}^{269}-(3 \mathrm{mts}){ }_{104} \mathrm{Rf}^{265}$

${ }_{96} \mathrm{Cm}^{248}-116^{292}$, which lasts for $50-125$ millisec then decays to- $114^{288}$.

(b) Nomenclature: The IUPAC, had accepted their existence in 2005 but their names were officially decided in November 2011. The '114' has been named Flerovium(Fl) to honor Gregory.N. Flerov, a nuclear scientist at JINR, Russia and '116' is given the name Livermorium(Lv), in honor of the city of Livermore, where the LLNL,U.S.A is situated. These names were officially published in July, 2012.

(c) Position in table; The electronic configuration of $\mathrm{Fl}$ and $\mathrm{Lv}$ are;

$[\mathrm{Rn}]-5 \mathrm{f}^{14} 6 \mathrm{~d}^{10} 7 \mathrm{~s}^{2} 7 \mathrm{p}^{2}$

$[\mathrm{Rn}]-5 \mathrm{f}^{14} 6 \mathrm{~d}^{10} 7 \mathrm{~s}^{2} 7 \mathrm{p}^{4}$

These elements are also in seventh period, both are members of p-block but former shows metallic character. Fl is in $14^{\text {th }}$ group and is called 'ekalead' and Lv, is in $16^{\text {th }}$ group and appears to be metalloid like Po, but as very little of the element has been made it is difficult to confirm it's properties.

\section{(v) Elements 117 \& 118:}

The 'E117', is a superheavy, artificial and $2^{\text {nd }}$ heaviest element. In 2008, it's discovery was announced jointly by LLNL,U.S.A and JINR, Dubna and synthesis was repeated in April, 2010, by smashing together Bk target with $\mathrm{Ca}$ beam as shown;

${ }_{97} \mathrm{Bk}^{249}+{ }_{20} 20 \mathrm{Ca}^{48}-117^{297}+3{ }^{1} \mathrm{n}_{0}$

The experiment was repeated in 2011, but was unsuccessful. The IUPAC, does not reconise it's existence.

The 'E118' is the only synthetic member of group 18, has highest atomic mass, and is very unstable. In 1998, a Polish scientist Robert Smolanczuk, suggested the fusion of $\mathrm{Pb}$ and $\mathrm{Cr}$ for it's preparation. In 2002, LBNL, using this reaction, predicted ,that the element could be made as; ${ }_{36} \mathrm{Kr}^{86}+{ }_{82} \mathrm{~Pb}^{208}-118^{293}$

This claim was rejected on October 19 2006, by IUPAC.

In the same year, a joint collaboration of LLNL and JINR, repeated the experiment using Cf and Ca and claimed to have prepared 3 nuclei of element as; ${ }_{98} \mathrm{Cf}^{249}+{ }_{20} \mathrm{Ca}^{48}-118^{294}+3^{1} \mathrm{n}_{0}$

IN 2011, IUPAC reported that the results do not satisfy the criterian of discovery. The authors do not agree that their results are false and have verified the existence of $119^{294}$ in a reaction of type;

${ }_{96} \mathrm{Cf} 245+{ }_{20} \mathrm{Ca} 48-118^{294}$, this has been identified by it's decay chain as shown;

$118^{294}-{ }_{116} \mathrm{Lv}^{290}-$ (14millisec) $-{ }_{114} \mathrm{Fl}^{286}$-(alpha decay) $-{ }_{112} \mathrm{Cn}^{282}-$ Spontaneous fission

(b) Nomenclature; No name has yet been given by IUPAC.

(c) Position in table; The element is in seventh period, is in p-block and belongs to $18^{\text {th }}$ group. It's configuration is; [Rn] $5 \mathrm{f}^{14} 6 \mathrm{~d}^{10} 7 \mathrm{~s}^{2} 7 \mathrm{p}^{6}$.

It should be inert in it's properties and is called 'eka-Radon' for the time being, till an official name is given by IUPAC.

\section{(4) Elements 119 \& 120}

' 119 '

After the announcement of elements till $Z=118$, the race for preparing the ' 119 ' started at Oak Ridge National Laboratory, U.S.A, by fusion of Bk (a perishable element with a half life of 320 days) with Ti, as shown; ${ }_{22} \mathrm{Ti}+{ }_{97} \mathrm{Bk}-$ ' 119 '.

It is very difficult to create intense $\mathrm{Ti}$ beam, moreover the only way to detect the element, is by detecting daughter nuclei, which has not been possible.

' 120 '

Synthesis of ' 120 ', was attempted in November 2011, at the institute at Darmstadt, by;

${ }_{98} \mathrm{Cf}+22{ }_{22} \mathrm{Ti}-$ ' 120 ', 
but the attempt has been unsuccessful.

While concluding the article, it can be said, although 118 elements are listed in P.T, only 112, have been recognized by IUPAC. But scientists are of view, that, 'Ununoctium' is not the end of the table and are determined to go on trying till they are successful further . 